\title{
Alcohol Reactions in Subjects of European Descent: Effects on Alcohol Use and on Physical and Psychomotor Responses to Alcohol
}

\author{
J. B. Whitfield and N. G. Martin
}

\begin{abstract}
Self-reports of reactions to small amounts of alcohol, obtained between 1990 and 1992, were compared with reports of alcohol use, obtained in 1990-1992 and also in 1979-1981, in twin subjects of European descent Data on subjective, physiological, psychomotor, and metabolic responses to a test dose of alcohol, taken in 19791981, were also available. Alcohol reactions were more common in women than in men, and were associated with less alcohol use, both at the time that information about reactions was obtained and as recorded on average 12 years previously, in both sexes. Physiological and psychomotor responses to alcohol were similar across the reaction groups, except that deterioration in standing steadiness was greater in those who subsequently reported adverse reactions to alcohol. Contrary to expectation, skin temperature changes after alcohol were less in the subjects who reported ahways reacting to alcohol than in the other groups. Subjective reports of intoxication were greatest in subjects who subsequently reported alcohol reactions. The pattem of twin pair concordance for reactions suggests low heritability, so alcohol reactions in subjects of European descent are not caused by a single gene of high penetrance of the type found in the Asian alcohol flush reaction.
\end{abstract}

Key Words: Alcohol Flush Reaction, Europeans, Alcohol Consumption, Skin Temperature, Alcohol Dependence.

$\mathbf{T}$ HE ALCOHOL flush reaction in Japanese, Chinese, and some other Asian subjects is a well-studied phenomenon that represents a unique example of a single gene having a major effect on alcohol use and alcohol dependence. $^{1-3}$ This inherited aldehyde dehydrogenase-2 $(\mathrm{ALDH} 2)$ deficiency is rare in non-Asian subjects; although similar reactions occur in some North and South American native groups they seem to be from a different enzyme defect. ${ }^{4}$ Reactions to alcohol in European-descent groups have attracted little attention and are, in the majority of cases, of unknown cause.

We have previousiy reported that acute reactions to

From the Department of Clinical Biochemistry (J.B.W.), Roval Prince Alfred Hospital Camperdown. NSW, Australia; and Queensiand Instirute for Medical Research (N.G.M.), Brisbane, Queensland Australia.

Received for publicaiion November 14, 1994; accepted August 30, 1995

The alcohol challenge twin study, and pilot studies of follow-up of the subjects, were suppored by the Australian Associated Brewers. The follow-up study and genotyping were supponed by the National Health and Medical Research Council Continuing contact with the subjects has been maintained through the Austraiian NHMRC Twin Registry and with the aid of the National Institute on Alconol Abuse and Alcoholism Grant AA07535.

Reprint requests: J. B. Whitfield. Ph.D.. Department of Clinical Biochemistry, Roval Prince Alfred Hospital. Camperdown. NSW 2050. Australia.

Copvright (C) 1996 by The Research Socien on Alconolism. small amounts of alcohol occur in $\sim 5 \%$ of subjects of European descent, and that they have an aversive effect in that subjects reporting them also report lower alcohol consumption on a number of measures than nonreacting subjects. ${ }^{5}$ This may be a discrete metabolic phenomenon, like the Asian alcohol flush reaction caused by ALDH deficiency, but there is a need to define its effects more closely and to determine whether it shows the strong pattern of heritability to be expected if it is caused by an enzyme deficiency.

We have now completed a study of twin subjects previously tested with alcohol. This article describes the associations between self-reported alcohol reactions and alcohol consumption at different times in the subjects' lives, and symptoms of alcohol dependence. We have also tested whether physiological. subjective, and psychomotor responses to alcohol differ between European subjects who report reactions and those who do not. The concordance of twin pairs has been used to determine whether there is evidence for an inherited basis for European alcohol reactions.

\section{SUBJECTS AND METHODS}

Three hundred and thirty-four subjects (157 males, 177 females) provided answers in 1990-1992 to a questionnaire containing sections on unpieasant reactions to small amounts of alcohol, alcohol use, and lifetime experience of symptoms or events associated with alcohol dependence. They were aged $27-46$ years (mean $=34.1, S D=4.8$ years) at the time of participation and had aiso taken part, 9-13 years previously (1979-1981), in a study of alcohol metabolism and susceptibility to intoxication. ${ }^{6.7}$

All subjects were twins, but the number of complete pairs is rather less than half the number of subjects because it was not possible to contact or include both twins in every case. There were 29 pairs of monozygotic (MZ) and 25 pairs of dizygotic (DZ) male twins; 33 pairs of $M Z$ and 33 pairs of $D Z$ female twins: and 28 pairs of $D Z$ twins of the opposite sex.

\section{Questionnaire}

All subjects completed a questionnaire with questions about reactions to small amounts of alcohol, quantity and frequency of alcohol use, number of drinks in the previous week. and lifetime occurrence of symptoms associated with alcohol use or dependence. The questions were:

Do you experience unpleasant reactions, such as flushing of the face or body, itching, drowsiness. or palpitations after drinking a small amount of alcohol (e.g., one or two drinks):

Always

Sometimes

Never 
Please write in below the number that best describes hou often (you) have had alcoholic irinks during the lasi 12 months.

Write the number that best describes how many drinks (you) usually have in a typical week.

On the chart beiow. please write the number of drinks vou had on each day in the past week.

On average how many drinks would you have on each day that vou have some alcohol?

Was there ever a time when:

You drank too much

You felt guilty about drinking

Someone else objected to your drinking

You were treated for a drinking problem

You deliberately tried to cut down on your drinking, but were unable to jo so

You planned to stop drinking completely, but then failed to stick to vour plan

You got into physical fights while drinking

You went on binges where you kept drinking for a couple of days or more without sobering up

You went on drinking binges and neglected some of your usual responsibilities

You got into trouble driving an automobile after drinking

Was there ever a time when your drinking had a harmful effect on:

Your friendships and social life

Your health

Your marriage or home life

Your work or employment opportunities

Have you ever had a drink first thing in the morning to steady your nerves or to get rid of a hangover?

Have you ever had drinking problems lasting at least a month?

A quantity-frequency score for alcohol use was calculated by multiplying the number of days of alcohol use per week and the number of drinks usually taken on a drinking day. A score for alcohol dependence was calculated by adding the number of positive answers to the dependencerelated questions.

Data were also available for similar questions about aicohol consumption. but not alcohol reactions or alcohol dependence, which the subjects had answered some 12 years previousiy (in 1979-1981). Intake variables from that occasion were the usual number of drinks in a week and the quantity-frequency measure.

\section{Alcohol Challenge Study}

All subjects had taken part in an alcohol study, designed to investigate heritability of alcohol metabolism and intoxication. in 1979-1981. The amount of alcohol taken in that study was determined by the subjects' weight: $0.75 \mathrm{~g}$ of ethanol $/ \mathrm{kg}$. Baseline and postaicohol measurements of skin temperature, heart rate and blood pressure, performance on psychomotor tests, and subjective scores of intoxication had been recorded.6.7 and were available for analysis for effects of self-reported alcohol reaction status. Before drinking alcohol, the subjects had answered a questionnaire that included items on number of drinks taken per week. by rype of drink and their usual frequency and quantity of consumption. Skin temperature was measured by a thermistor probe taped to the cheek, and body sway was measured by the subject standing on a platform with a transducer that measured movement of the platform. Results reported in this article were obtained with the subjects' eves open. Two questions were used to assess subjective perceptions of intoxication: they reported on their intoxication on a scale of $1-10$ with $1=$ completely sober and $10=$ "The most drunk
I have ever been." And. they gave a yes/no answer to the question. "Wouid you drive a car now?" (yes was scored 1 and no was scored 0 ).

All of the subjects in the current study had been abie to drink :ne aicohol in 1079-1981. over a period of $20 \mathrm{~min}$. An unknown but smail number of other volunteers had been unable to drink the aicohol. or nad vomited. and no information from the initial study or follow-up is availabie for them.

\section{Zygosity Determination}

Details are given in Rei. 6. Twin pairs were classified as DZ if the twins were of the opposite sex. had differing types on blood grouping or plasma protein phenotyping, or differed markedly in height. natural hair type. or eye coloring. Otherwise. they were classified as $\mathrm{MZ}$.

\section{ADH Genotyping}

Blood samples were available from 327 of the subjects who completed the follow-up questionnaire: 154 men and 173 women. Alcohol dehydrogenase $(\mathrm{ADH})-2$ and $\mathrm{ADH} 3$ genotypes were determined on DNA extracted from white blood cells. Appropriate sections of DNA. containing the areas that differ between $A D H 2-1$ and $A D H 2-2$ and berween $A D H 3-1$ and $A D H 3-2$, were amplified by polymerase chain reaction using primers specific for $A D H 2$ or $A D H 3$. The primers used were: for ADH2 - (5')ATTCTGTAGATGGTGGCTGT and (5')GCCTAAAAT. CACAGGAAGG; for ADH3-(5')CTTTAAGAGTAAAGAATCT and (5')CTTTCCAGAGCGAAGCAGGTC. After amplification, the ADH2 product was incubated with MaeIII that cuts the $A D H 2-2$ sequence but not the $A D H 2-1$, whereas the $A D H 3$ product was incubated with SspI that cuts only $\mathrm{ADH} 3-1 .^{8.9}$ The digestion products were separated by electrophoresis and visualized with ethidium bromide to assign genotypes to the samples. Samples were also tested for $A D H 2-3$ by allele-specific amplisication. but no occurrences of $\mathrm{ADH} 2-3$ were found.

\section{Statistical Methods}

Subjects were ciassified into three groups (Always, Sometimes. or Never reacting) according to their answer to the question about alcoinoi reactions. Measured physiological. psychomotor, and subjective variables were tested for differences among reaction groups by anaivsis of variance. Alcohol use measures. and dependence scores. were transformed to $\log (x$ +1 ) scores to reduce skewness; the constant value of 1 was added to allow: subjects with 0 scores to be included. Tests for differences in the prevalence of reactions between men and women, and for association of alconoi reactions with $\mathrm{ADH}$ genotypes, were performed by Fisher's exact test using StatXact Turbo 2.1 (Cytel Software Corporation. Cambridge. MA).

\section{RESULTS}

\section{Prevalence}

The proportion of men and women responding Always. Sometimes, or Never to the question about alcohol reactions is shown in Table 1. The prevalence of reactions differed significantly between men and women $(p=0.0001$. Fisher's exact test). Because the prevalence of aicohol reactions, and the mean values for alcohol intake variables.

Table 1. Reported Prevalence of Reactions in Males and Females

\begin{tabular}{lcl}
\hline & Males & Females \\
\hline Always & $6(3.8 \%)$ & $12(6.8 \%)$ \\
Sometimes & $30(19.1 \%)$ & $67(37.9 \%)$ \\
Never & $121(77.1 \%)$ & $98(55.4 \%)$ \\
\hline
\end{tabular}

Fisher's exact test. $p=0.0001$ 


\section{$\log ($ drinks +1$)$}

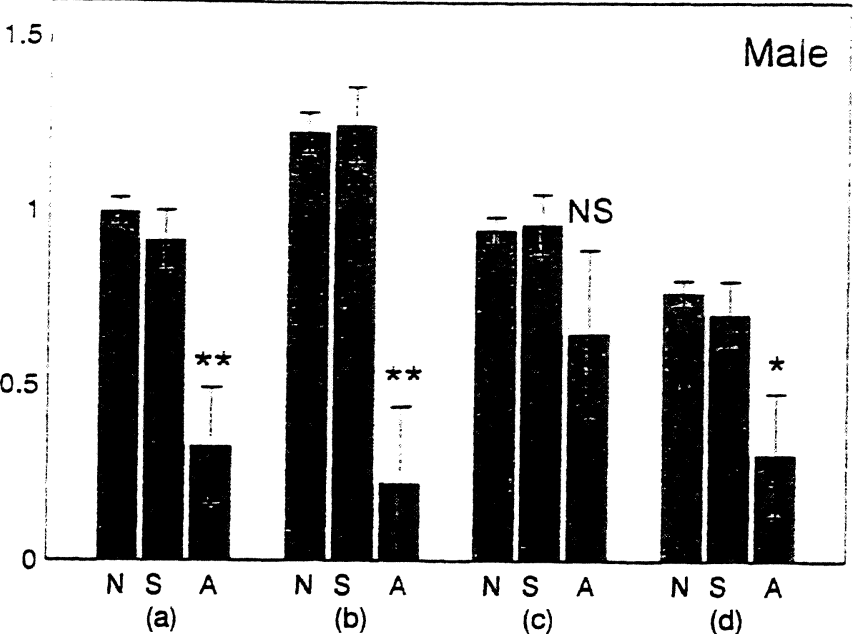

$\log ($ drinks +1$)$

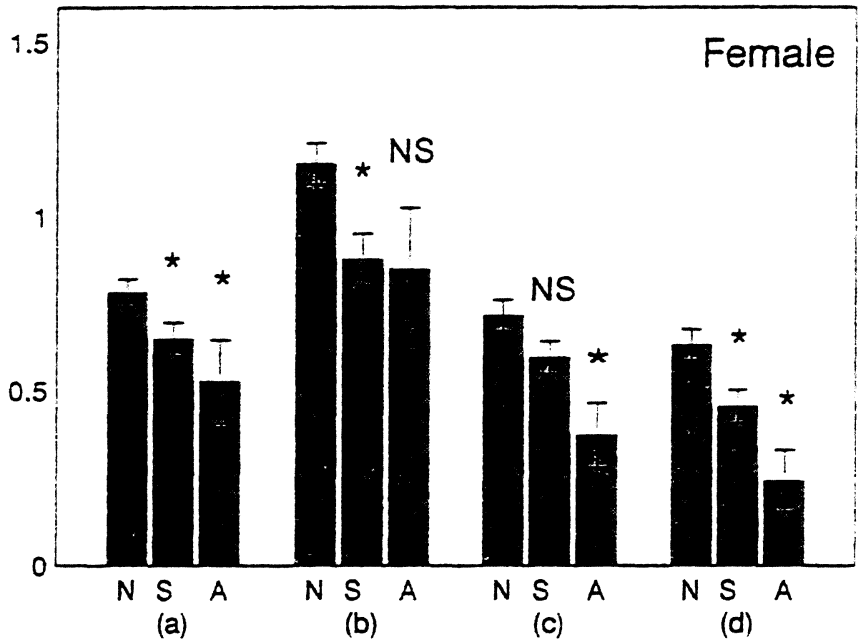

Fig. 1. Effects of alcohol reactions on alconol intake in men and women. Bars show the mean log-transtormed weekly alcohol intake in standard drinks. with SEM, categorized by reaction status $(A=$ always. $S=$ sometimes, and $N=$ never). (a) Weekly drinks in 1979-1981. (b) quantity-frequency measure 19791981. (c) weekly drinks in 1990-1992, and (d) quantity-trequency measure 19901992. Significance of mean difference from the Never group is shown as: NS, not significant; $\cdot p<0.05: \cdots p<0.01$.

differed between men and women, separate analyses of male and female results have been performed. Where no significant effect of reaction has been found for either sex, data for both sexes have been combined in case the lack of significance is caused by small numbers of subjects in the Always reacting group.

\section{Alcohol Use}

Effects of alcohol reaction group on several measures of alcohol use are shown in Fig. 1. Alcohol intake measures were available at two times: at the time of the questionnaire (i.e., answers about reactions are contemporary with the answers about alcohol use) and 12 years previousiy (i.e.. alcohol use recorded in 1979-1981 and reaction informa- tion provided later). The measures are of two types, basec on number of drinks in a week or on quantity and frequency measures. and are shown separately for men and women. Alcohol reactions were associated with lower alcohol use. and their effect extended across the two occasions of questioning about alcohol use, some 10 years apart. Lifetime alconol dependence scores did not differ significantly between the reaction groups, but there were few subjects with high scores (especially among the women) and few men who reported reactions.

\section{Alcohol Metabolism}

There was no significant association between blood alcohol concentrations, peak blood alcohol concentration. rate of decline, and the prevalence of reactions. The relationship between reactions and $\mathrm{ADH} 2$ or $\mathrm{ADH} 3$ type is not clear-cut: data are shown in Table 2. For ADH2 type, there was a significant association ( $p=0.037$, Fisher's exact test), but the cell frequencies are low. Whereas the response Always is more likely in the $\mathrm{ADH} 2-1,2$ subjects, the response Sometimes is more common in the $\mathrm{ADH} 2-1,1$ group. ADH3 type was not significantly associated with alcohol reactions $(p=0.538)$.

\section{Response to Experimental Alcohol Consumption}

A number of physiological reactions to alcohol were measured in the 1979-1981 alcohol challenge study. For skin temperature, there were significant differences be tween alcohol reaction groups at times 1 and 2, but not at time 3 nor at (prealcohol) time 0 . The Always group was significantiy different from both the Sometimes group ( $p<$ $0.05)$, and the Never group $(p<0.01)$ at times 1 and 2 . Skin temperature readings for the Sometimes and Never groups were essentially identical but, surprisingiy, the Always group had the lowest temperature increase after alcohol.

For body sway, a marginally significant difference between groups $(p=0.059)$ was found at time 1 . However, when the change in body sway between time 0 (preaicohol) and time 1 was calculated, there was a highly significant difference between the groups $\left(F_{2331}=7.29, p<0.001\right)$. The increase in body sway in the Always group was significantly greater than for the Sometimes group $(p<0.05)$ or the Never group $(p<0.01)$; once again, the last two groups showed very similar results.

Table 2 Subjects' Responses to Alconol Reaction Question. by ADH2 anc ADH3 Types

\begin{tabular}{lcrr}
\hline & Always & Sometimes & \multicolumn{1}{c}{ Never } \\
\hline ADH2-1.1 & $14(4.7 \%)$ & $90(30.2 \%)$ & $194(65.1 \%$ \\
ADH2-1.2 & $4(13.8 \%)$ & $4(13.8 \%)$ & $21(72.4 \%$ \\
$D=0.037$. Fisher's exact test & & & \\
& & & \\
ADH3-1.: & $4(4.4 \%)$ & $24(26.4 \%)$ & $53(69.2 \%)$ \\
ADH3-i.2 & $9(4.9 \%)$ & $52(28.4 \%)$ & $i 22(66.7 \%)$ \\
ADH3-2.2 & $5(9.6 \%)$ & $17(32.7 \%)$ & $30(57.7 \%)$ \\
$D=0.538$. Fisner's exact test & & & \\
\hline
\end{tabular}



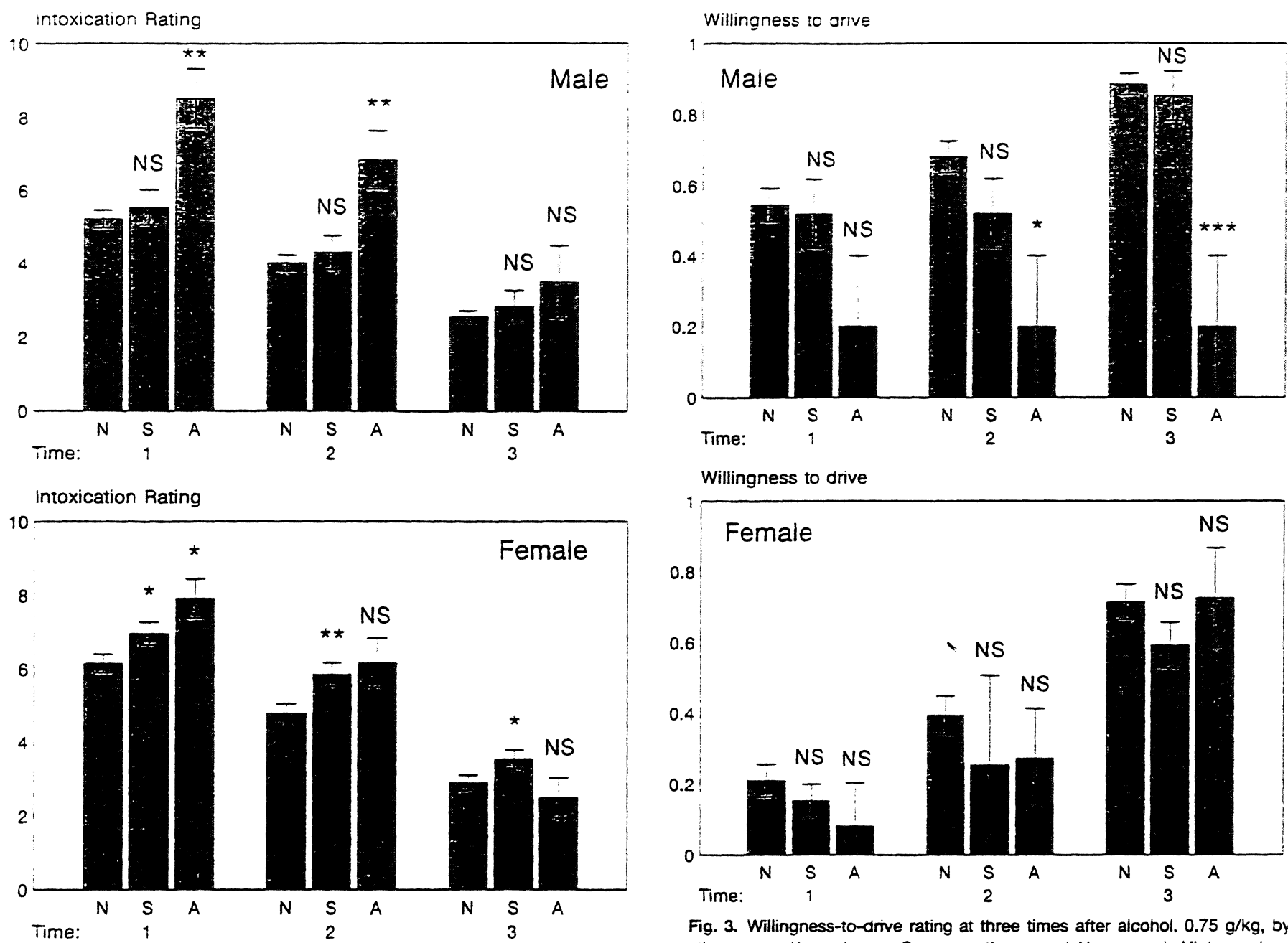

Time:

Fig. 3. Willingness-to-drive rating at three times after alcohol. $0.75 \mathrm{~g} / \mathrm{kg}$, by reaction status ( $A=$ always. $S=$ sometimes, and $N=$ never). Higher values indicate greater willingness to drive. Significance of mean difference trom the Never group is shown as: NS, not significant, $p<0.05 ;-p<0.01$.

reaction status $(A=$ always, $S=$ sometimes, and $N=$ never). Higher values indicate greater perceived intoxication. Significance of mean difference from the Never group is shown as: NS, not significant: ${ }^{*} p<0.05:{ }^{*} p<0.01$.

There were no significant differences (i.e., all $p>0.05$ ) for pulse rate, or systolic and diastolic blood pressures, between the alcohol reaction groups. Alcohol's effects on psychomotor test abilities, including reaction time, tracking ability, and divided attention tasks, did not differ significantly according to reaction group in either males or females, nor when results from both were taken together.

Effects of reported reactions on subjective impressions of intoxication, and on willingness to drive, showed several signinicant differences. The results for these two questions are shown in Figs. 2 and 3. There were significant effects of alcohol reactions on perceived intoxication at postalcohol times 1 and 2, but not time 3 , for both men and women. For men. the main difference was between those replying Always and those replying either Sometimes or Never, whereas for women the results for the Sometimes group were intermediate and significantly different from the Never group. For the willingness-to-drive question, reactions had little effect in women (who tended in any case to be more cautious than men in their response). In men, they

had a significant effect at the later times (postalcohol times 2 and 3) and once again only consistent ("Always") reactions influenced the response. The Sometimes group showed negligible differences from the unaffected ("Never") group.

\section{Twin Concordance for Reported Alcohol Reactions}

The pair concordances for reported alcohol reactions are calculated as polychoric correlations: $M Z$ males $=-0.02$, $\mathrm{MZ}$ females $=0.39 ; \mathrm{DZ}$ males $=-0.08, \mathrm{DZ}$ females $=$ -0.34 ; and opposite-sex $\mathrm{DZ}$ pairs $=-0.06$. None of these are significantly different from 0 . It was found that of 16 subjects who replied Aiways to the alcohol reaction question and whose cotwin's response is also known, none had an Always reacting twin (regardless of zygosity) and more of their cotwins replied Never (11 subjects) than Sometimes (5 subjects)..$^{5}$

\section{DISCUSSION}

We would expect that people who find aicohol use unpleasant because of acute reactions to small amounts would 
drink less than others and would therefore be less likely to abuse alcohol or become dependent. This has been confirmed in numerous studies on Japanese and Chinese subjects. As previousiy reported. ${ }^{5}$ aicohol reactions affect alcohol use in European as well as Asian groups; but the nature and causes of alcohol reactions in non-Asian populations are not well documented. This article considers their effects on drinking habits as assessed at different times and their effects on signs of alcohol dependence, and relates self-reported reactions to the subjects' physiological, psychomotor, and subjective responses to an experimental dose of alcohol.

Reactions to alcohol, which the subjects considered unpleasant. were reported to occur on at least some occasions of drinking by almost one-quarter of the men and almost one-half of the women (Table 1). The experience of reacting every time alcohol is used is much less common, at $\sim 4 \%$ in men and $7 \%$ in women. A broadly similar incidence and sex difference has been reported by Ward et al. ${ }^{10}$ from a questionnaire on flushing reactions that was completed by 18- to 24-year-old students in London. These significant differences in reaction rates between men and women are of unknown cause, and may reflect either differences in perception or in willingness to report reactions, or true differences in the physiological response to aicohol.

Although the prevalence of alconol reactions is lower in subjects of European descent than in some Asian groups, they still exert a significant influence on drinking habits. This is true for both men and women, even though reactions are more commonly experienced or reported by women. There is evidence that the reactions persist over time and have a lasting effect. because those who reported the reactions had also previously reported lower alcohol intake 12 years before the current study, as well as at the time of questioning about reactions (Fig. 1). There was no significant effect on symptoms of aicohol dependence, but the number of subjects was probably too small to detect any effect; few of the subjects reported aiways reacting to alcohol and the incidence of alcohol dependence was low.

These findings on alcohol use contrast with those in the London study previously cited, wherein no effect on alcohol consumption could be demonstrated. The reasons for this difference may lie in the age and composition of the London sample, who were all medical students; even among ALDH2 heterozygotes in Japan, social pressure to drink is gradually increasing the proportion who use or abuse alcohol. ${ }^{11}$ Another difference, which may be important, is that the question used in the London study did not distinguish between reactions occurring every time alcohol is taken and reactions that only occur sometimes. In our subjects, it was the reactions occurring every time that had the greatest effect on alcohol use, particularly for men.

The reactions in Europeans show a number of clear differences from those in Asians. The existence of $M Z$ twin pairs discordant for self-reported reactions shows that European alcohol reactions are not soleiv caused by a mono- genic inborn error of metabolism, such as the well-explored Asian ALDH2 deficiency causing the aicohol flush reaction. ALDH deficiencies have been documented in a few subjects of European descent who experience alcohol reactions, ${ }^{10.12}$ but there are few well-studied exampies and such enzyme deficiency seems to be an uncommon cause of alcohol reactions outside Asian groups.

A significant association between $\mathrm{ADH} 2$ type and the occurrence of reactions was found, but data suggest that this is a secondary factor, perhaps influencing the reaction enough to move some subjects from the "Sometimes" caiegory to the "Always" category (Table 2). Larger numbers of subjects would be needed to establish any $A D H 2$ genotype effect in Europeans. However, it is of interest to note that $\mathrm{ADH} 2$ type, as well as ALDH2 type, affects the aicohol flush reaction in Asians. Thomasson et al. ${ }^{13}$ reported that. among Chinese subjects with normal ALDH2 activity, ADH2-2 homozygotes had a greater increase in facial blood flow after alcohol than $\mathrm{ADH} 2-1,2$ heterozygotes. Data for ADH2-1 homozygotes was not reported; presumably there were insufficient subjects of this genotype in the group studied.

The physiological changes produced by alcohol are less than those reported in Asians, with no significant differences in pulse rate, blood pressures, or most tests of psychomotor performance between the reaction groups. Significant differences were found in facial skin temperatures, which would be expected to be the most prominent feature of flushing reactions, but these differences were in the opposite direction to what would be expected; those who reported "Always" reacting to alcohol had smaller skin temperature increases after alcohol. This also is in contrast with the results of Ward et al. ${ }^{10}$; they measured blood flow by a Doppler method and showed an increase in European flushers over nonflushers, although it was much smaller than the increase seen in an Oriental flusher.

Subjective assessments of intoxication after a test dose of alcohol were greater in the subjects who subsequently reported reactions. This was true both for the question about degree of intoxication (Fig. 2) and for the subjects' expressed willingness to drive a car (Fig. 3). There was also a significant difference in body sway between the subjects who reported reactions "Alwavs" and those who did not, with a greater change after alcohol in those who reported alcohol reactions. Body sway, experienced as a feeling of unsteadiness, may well be one of the factors affecting subjects' perception of their degree of intoxication; it correlates significantly with self-report intoxication in both men and women in this sample even after controlling for the reaction responses (data not shown), and it is one of the aspects of the experience of intoxication claimed to influence subsequent aicohol dependence risk. ${ }^{14}$

Perception and report of reactions are likely to be influenced by both physiological and psychological characteristics. People who drink little, for whatever reason. might have negative expectations about alcohol's effects and 
therefore interpret their responses as unpleasant. However. average weekly alcohol consumption shows substantial heritability in both women and men ${ }^{15}$ and if the alcohol reactions (or the subjects' perceptions of them) are determined by alcohol consumption, then we would expect evidence of heritability for the reported reactions. This was not the case.

The way that these reactions are acquired, and the way that alcohol produces them, are still unknown. There may well be a number of causes for the self-reported reactions to alcohol in Europeans, including metabolic, physiological, and immunological (allergic) components. Some of the subjects may be found to have an inherited biochemical cause for their reactions, but these will be a minority among European subjects with alcohol reactions. Further exploration of the range of symptoms experienced in a much larger group of twin subjects, which is in progress, should shed light on heterogeneity of alcohol reactions.

\section{REFERENCES}

1. Wolff PH: Ethnic differences in alcohol sensitivity. Science 175 : $449-450,1972$

2. Goedde HW. Harada S. Agarwal DP: Racial differences in alcohol sensitivity: A new hypothesis. Hum Genet 51:331-334. 1979

3. Crabb DW. Edenberg HI, Bosron WF. Li T-K: Genotypes for aldehyde dehydrogenase deficiency and alcohol sensitivity. J Clin Invesi 83:314-316, 1989

4. O'Dowd BF, Rothhammer F, Israel Y: Genotyping of mitochon- drial aldehvde dehvarogenase locus of native American Indians. Alcohoiism 14:531-533, 1990

5. Whitfield JB. Martin NG: Aversive reactions and alcohol use in Europeans. Alcohol Cin Exp Res 17:13:-134, 1993

6. Martin NG. Perl J, Oakeshon JG. Gibson JB. Starmer GA. Wilks AV: A twin study of ethanol metabolism. Behav Genet 15:93-109, 1985

7. Martin NG, Oakeshot JG, Gibson JB, Starmer GA, Perl J, Wilks AV: A twin study of psychomotor and physiological response to an acute dose of alcohol. Behav Genet 15:305-347, 1985

8. von Wartburg JP, Gennari K, Muellener D, Wermuth B: Determjnation of the genotype of human class I alcohol dehydrogenase, in Kuriyama K, Takada A. Ishii H (eds): Biomedical and Social Aspects of Alcohol and Alcoholism. Amsterdam. Excerpta Medica. 1988, pp 35-38

9. Xu Y, Carr LG. Bosron WF, Li T-K. Edenberg HJ: Genotyping of human alcohol dehydrogenases at the $\mathrm{ADH} 2$ and $\mathrm{ADH} 3$ loci foliowing DNA sequence amplification. Genomies 2:209-214, 1988

10. Ward RJ, McPherson AJS. Chow C, Ealing J, Sherman DIN. Yoshida A. Peters TJ: Identification and characterisation of alcoholinduced flushing in Caucasian subjects. Alcohol Alcohol 29:433-438, 1994

11. Higuchi S: Polymorphisms of alcohol metabolizing enzyme genes and alcoholism. Alcohol Clin Exp Res 18:8A, 1993 (abstr)

12. Yoshida A. Dave V, Ward RJ. Peters TJ: Crosolic aldehyde dehydrogenase (ALDH1) variants founc in alcohol flushers. Ann Hum Genet 53:1-7, 1989

13. Thomasson HR, Crabb DW, Eoienberg HJ, Li T-K: Alcohol and aldehyde dehydrogenase polymorphisms and alcoholism. Behav Gene: 23:131-136, 1993

14. Schuckit MA: Reaction to alcohol as a predictor of alcoholism, in Taberner PV, Badawy AA (eds): Advances in Biomedical Alcohol Research. Oxford. Pergamon Press, 1993. pp 91-94

15. Heath AC, Marin NG: Genetic influences on alcohol consumption patterns and problem drinking: Results from the Australian NHMRC Twin Panel Follow-Up Survey. Ann NI Acad Sci 708:72-85, 1994 